\title{
Adaptación del Cuestionario de Competencia Interpersonal ICQ-15 con Población Adolescente Hispanohablante
}

\author{
Adapting the ICQ-15 Interpersonal Competence Questionnaire with an Adolescent \\ Spanish-Speaking Population
}

\author{
Carlos Salavera ${ }^{1,2}$ y Pablo Usán ${ }^{1,2}$
}

\begin{abstract}
Resumen
El objetivo de este estudio fue la adaptación y validación del Inventario de Competencia Interpersonal ICQ15 (Buhrmester, Furman, Wittenberg, \& Reiss, 1988), probado con adultos, al caso de los adolescentes hispanohablantes ( $N=1060$, edad $=16,23$ años). Se realizaron dos estudios: 1) se realizó la traducción del ICQ-15 al español y se evaluó su consistencia interna, estructura factorial y validez convergente; y 2) se realizó el análisis factorial confirmatorio del cuestionario. El análisis factorial exploratorio mostró claramente que los factores del ICQ-15 presentan una varianza acumulada del 64,88\%, mostrando ser capaces de explicar la variación única de competencia interpersonal en adolescentes. Por su parte, el análisis factorial confirmatorio confirma la adecuación del modelo, con un modelo sustentable compuesto por los cinco factores identificados y 15 ítems. El ICQ-15 es un instrumento fácil de comprender y rápido de completar, por lo que se considera útil para la evaluación de la competencia interpersonal en adolescentes.
\end{abstract}

Palabras clave: adolescentes, competencia interpersonal, evaluación, bienestar afectivo

\begin{abstract}
The objective of this study was to adapt and validate the ICQ-15 Interpersonal Competence Questionnaire (Buhrmester, Furman, Wittenberg, \& Reiss, 1988), which has been tested with adults, with Spanish-speaking adolescents $(N=1060$, age $=16.23$ years). Two studies were conducted: 1$)$ the ICQ-15 was translated into Spanish, and its internal consistency, factorial structure and convergent validity were evaluated; 2) a confirmatory factorial analysis of the questionnaire was done. The exploratory factorial analysis clearly showed that the ICQ-15 factors presented an accumulated variance of $64.88 \%$, and proved capable of explaining single variation for interpersonal competence in adolescents. Moreover, the confirmatory factorial analysis confirmed the suitability of the model, with a viable model that comprised the five identified factors and 15 items. As the ICQ-15 is an easy instrument to understand and quick to complete, it is considered useful to evaluate interpersonal competence in adolescents.
\end{abstract}

Keywords: adolescents, interpersonal competence, evaluation, affective well-being

\footnotetext{
${ }^{1}$ OPIICS Observatorio para la y Investigación en Ciencias Sociales, Universidad de Zaragoza, C/Pedro Cerbuna, 12 , 50009 Zaragoza, España. Correo: salavera@unizar.es

${ }^{2}$ Facultad de Educación, Universidad de Zaragoza, C/Pedro Cerbuna, 12, 50009 Zaragoza, España.
} 


\section{Introducción}

Los adolescentes tienen como un punto fundamental en su proceso madurativo, el desarrollo de las relaciones con sus iguales, cuestión que aún con lasambigüedades en su bienestar afectivo en esta época de su vida, se torna casi indispensable no sólo para su propio proceso intrapersonal, sino también para abordar su necesidad de autonomía. Es lo que se ha venido denominando habilidades sociales, definidas como un conjunto de conductas necesarias para interactuar y relacionarse con los demás de una manera efectiva y satisfactoria, sirviendo para mostrar la capacidad para comportarse de una forma que es recompensada y evitar comportarse de forma que sea castigado o ignorado por los demás (Kinnaman \& Bellack, 2012). Es decir, estas habilidades son un conjunto de conductas emitidas por el individuo en un contexto interpersonal que le sirve para expresar sus sentimientos, actitudes, deseos, opiniones o derechos de un modo adecuado a la situación, respetando esas conductas en los demás y que, generalmente resuelve los problemas inmediatos de la situación mientras minimiza la probabilidad de futuros problemas (Caballo, 2002). Se adquieren principalmente a través del aprendizaje por observación, imitación, ensayo e información, resultando ser conductas aprendidas. La persona no nace con un repertorio determinado de habilidades sociales, sino que la persona van incorporándolas a lo largo de su desarrollo, formación y crecimiento, existiendo dos tipos de habilidades sociales: básicas y complejas, siendo necesario el aprendizaje de las primeras para desarrollar las segundas. Este proceso se inicia en la infancia, desarrollándose fundamentalmente en la adolescencia para dotar a la persona de herramientas comunicativas y de relación en la edad adulta, viniendo a cubrir la necesidad de establecer unas relaciones sociales positivas como fuente de satisfacción y bienestar personal (Holopainen, Lappalainen, Junttila \& Savolainen, 2011; Körner, Drapeau\&Geyer, 2006; Segrin, 2001).

En 1988, Burmeister, Furman, Wittenberg\& Reis desarrollaron el cuestionario de Competencias Interpersonales (ICQ), del que más tarde se planteó una versión abreviada (ICQ-15).
Este instrumento se ha utilizado con adultos, población clínica, ... dadas las características de la prueba, se plantea la posibilidad de su utilidad con adolescentes españoles.

El Cuestionario de Competencia Interpersonal (ICQ; Buhrmester, Furman, Wittenberg, \& Reis, 1988) es un instrumento de 40 ítems que evalúa lacompetencia interpersonal en cinco apartados: 1) iniciación de relaciones y/o conversaciones (por ejemplo, "presentarse a alguien a quien te gustaría llegar a conocer o con quién te gustaría salir"; 2) aserción negativa (por ejemplo, "decirle a un conocido que ha hecho algo que te hizo enfadar"; 3) soporte emocional (por ejemplo, "ser capaz de aconsejar a un buen amigo cuando éste necesita ayuda y apoyo de modo que reciba bien los consejos"; 4) autorevelación (por ejemplo, "dejar que un nuevo compañero te conozca tal y como eres" y 5) gestión de conflictos (por ejemplo,ser capaz de ponerte en la posición de un compañero durante una discusión y entender su punto de vista). Los ítems se puntúan según una escala tipo Likert de 5 puntos, que varía desde 1 (me siento incómodo e incapaz de manejar esta situación) a 5 (me siento muy cómodo y podría manejar muy bien esta situación).

Aunque el ICQ fue desarrollado y validado con estudiantes universitarios, se ha demostrado su utilidad clínica con otras poblaciones: adolescentes con trastornos de la alimentación (Ferriter, Eberhart, \&Hammen, 2010) o adultos con trastornos de personalidad o ansiedad social (Muralidharan, Sheets, Madsen, Craighead, \& Craighead, 2011; Parade, Leerkes, \& Blankson, 2010).

El ICQ ha demostrado adecuadas propiedades psicométricas, tanto en su propuesta inicial (Buhrmester et al., 1988), como en las adaptaciones a otros idiomas y/o culturas (Kanning, 2006; Giromini, de Campora, Brusadelli, D’Onofrio, Zennaro et al., 2015) y en su forma abreviada, de 15 ítems (Coroiu, Meyer, Gomez-Garibello, Bräller, Hessel, \& Körner, 2015).

Aunque para otros constructos (autoconcepto, asertividad,...) se están desarrollando instrumentos de evaluación (Gálvez-Nieto, Polanco, \& Salvo, 2017; Navarro, Varas, \& Maluenda, 2017), son más escasos en el caso de la competencia social. En el caso del cuestionario de 
competencia social ICQ, explica la naturaleza de los distintos ámbitos de la competencia interpersonal y aclara los roles que desempeñan estos dominios en el funcionamiento social (Buhrmester et al., 1988). Esto, junto con la sencillez y rapidez de administración de esta escala de competencia interpersonal, motivó la traducción y validación de su forma abreviada (ICQ-15) con adolescentes al español, con la finalidad de dotar a la comunidad científica hispanohablante de un instrumento útil para la evaluación de su competencia social.

Los objetivos del presente estudio fueron dos: 1) evaluar la posibilidad de utilización del cuestionario de Competencia Interpersonal ICQ15 con adolescentes, como instrumento de evaluación de lacompetencia interpersonal en esas edades; y 2) la validación del ICQ-15 para hispanohablantes.En el estudio 1 se realizó la traducción del ICQ-15 al español y se evaluó su consistencia interna, estructura factorial y validez convergente. En el estudio 2 se realizó el análisis factorial confirmatorio del cuestionario.

La investigación presenta una única hipótesis de partida: el cuestionario de competencia interpersonal ICQ-15 es válido para evaluar este constructo en adolescentes.

\section{Método}

Para la adaptación del ICQ-15 al español se siguió el procedimiento clásico de traducción hacia atrás (Muñiz, Elosua, \& Hambleton, 2013), que comprende los siguientes pasos:

1. Partiendo de la versión original en inglés, se ha traducido al español por dos personas bilingües con lengua española de origen y con experiencia en literatura científica del ámbito de la evaluación psicológica. Ambas traducciones se discutieron con el equipo investigador, hasta alcanzar un consenso y generar la primera versión de la escala en español.

2. Un psicólogo con experiencia laboral en países de habla inglesa evaluó la equivalencia conceptual, la claridad y la naturalidad de cada una de las frases y opciones de respuesta de esta primera versión. Con las modificaciones oportunas, se obtuvo una segunda versión.

3. Se realizó una consulta de esta primera versión con psicólogos que trabajaban con adolescentes.
4. La segunda versión en español, se ha retrotraducido de nuevo al inglés por una traductora bilingüe, con lengua inglesa de origen.

5. Se realizó un prueba piloto con 50 alumnos/as de Secundaria, con la finalidad de evaluar la comprensión, tiempo necesario para la cumplimentación, claridad de las preguntas y adecuación de las respuestas.

6. Con el resultado de las consultas, el equipo investigador, realizó una tercera versión del ICQ15 y se aplicó para evaluar su aplicabilidad a población adolescente española.

\section{Participantes}

La muestra de la investigación se conformó para los dos estudios con 1060 estudiantes de Secundaria (526 hombres y 534 mujeres), la edad media de los participantes, se situó en 16.23 años, con un rango entre los 12 y 17 años, con una desviación estándar de 2.203. En el primer estudio, participaron 429 sujetos (213 hombres y 216 mujeres) y en el segundo estudio 631 (313 hombres y 318 mujeres). La participación de todos los sujetos fue voluntaria, firmaron el consentimiento informado y se respetaron las normas éticas siguiendo la Declaración de Helsinki y cumplió los criterios éticos de investigación con seres humanos (consentimiento informado de padres y madres, profesores y alumnos y derecho de información, protección de datos personales y garantías de confidencialidad, no discriminación, gratuidad y posibilidad de abandonar el estudio en cualquiera de sus fases). Los resultados se analizaron de manera anónima. Realizado el cálculo de la representatividad de la muestra, con un nivel de confianza del $99 \%$ y un error muestral del 5\%, se obtuvo que la muestra final encuestada resultó representativa de la provincia de Zaragoza (España). El estudio se diseñó como un estudio retrospectivo ex-post facto (Montero \& León, 2007).

Tabla 1. Distribución de la muestra por edades

\begin{tabular}{cccccc}
\multicolumn{6}{c}{$(\mathrm{n}=1060)$} \\
\hline \multicolumn{2}{c}{ Edad } & Frecuencia & Porcentaje & Edad & Frecuencia \\
\hline 12 & 166 & $15.66 \%$ & 15 & 266 & $25.09 \%$ \\
13 & 263 & $24.81 \%$ & 16 & 79 & $7.45 \%$ \\
14 & 267 & $25.19 \%$ & 17 & 19 & $1.79 \%$ \\
\hline
\end{tabular}




\section{Instrumentos}

Cuestionario de Habilidades Sociales ICQ-15 (Coroiu, Meyer, Gomez-Garibello, Brähler, Hessel, \& Körner, 2015), forma abreviada del ICQ (Buhrmester, Furman, Wittenberg, \& Reis, 1988).

Evalúa el constructo multimensional de las habilidades sociales a través de cinco escalas distintas, pero relacionadas entre sí, midiendo la competencia personal en la capacidad de: 1) iniciar relaciones; 2) aserción negativa; 3 ) revelar información personal; 4) proporcionar apoyo emocional; y 5) manejo de conflictos interpersonales. Consta de 15 ítems, de formato Likert de 5 puntos. Posee una fiabilidad alta $($ alfa $=.83)$. En la presente investigación, la fiabilidad de la escala fue de .85 .

Cuestionario Big-Five para niños y adolescentes (Barbaranelli, Caprara, Rabasca, \& Pastorelli, 2003), adaptado al castellano (Del Barrio, Carrasco, \& Holgado, 2006).

Este cuestionario, representa la adaptación del modelo de los Cinco Grandes de la personalidad para niños y adolescentes. Estas cinco dimensiones son: Conciencia (autonomía, orden, precisión, cumplimiento de normas y compromisos), Apertura (aspectos intelectuales, creatividad e intereses culturales), Extraversión (actividad, entusiasmo, asertividad $\mathrm{y}$ autoconfianza), Amabilidad (preocupación y sensibilidad hacia los otros y sus necesidades) e Inestabilidad emocional (ansiedad, depresión, descontento o ira). El sujeto debe contestar un total de 65 ítems en una escala tipo Likert de cinco puntos, con valores desde un punto hasta cinco puntos. En esta investigación se obtuvo una buena fiabilidad del cuestionario (alfa de Cronbach de .84).

\section{Diseño y procedimiento}

Para la selección de la muestra se solicitó la colaboración de los centros mediante llamada telefónica y, una vez confirmada la participación, se elaboró la lista de centros participantes. En el momento de la entrega de cada cuestionario, se procedió a explicar a los participantes el objetivo de la investigación, incidiendo en la importancia que tenía la cumplimentación de todos los ítems.
Los participantes tenían quince minutos para completar los cuestionarios que se acaban de describir. En todos los casos se les recordaba que la información recogida era anónima y confidencial.

Para el análisis estadístico de los datos se ha empleado el programa estadístico SPSS 22.0. Comprobada la normalidad de la muestra e igualdad de varianzas se optó por técnicas paramétricas.Se realizó un análisis descriptivo para cada una de las variables. En todos los casos se trabajó con el menor nivel de significación posible $y$ se han considerado significativas aquellas diferencias con un valor de $p<.05$. Los contrastes se plantearon de forma bilateral. Para el contraste de hipótesis de dos grupos se utilizó la $t$ de Student y para más de dos grupos la ANOVA unifactorial.Finalmente, con el programa estadístico AMOS v.24, se planteó un modelo de ecuaciones estructurales que permitiese validar y cuantificar relaciones causales entre los ítems y factores del ICQ-15. Siguiendo las recomendaciones de Batista y Coenders (2000) se ha utilizado el método de estimación de máxima verosimilitud y no el de mínimos cuadrados ponderados, dado el número de variables. Los datos recogidos en el presente estudio se obtuvieron entre los meses de abril y mayo de 2016.

\section{Resultados}

Los resultados se estructuraron según las dos etapas del proceso de investigación. Así, a continuación se describen los hallazgos del estudio 1: validez de constructo, análisis de consistencia interna, validez convergente y análisis factorial exploratorio (AFE); y del estudio 2, que comprende el análisis factorial confirmatorio (AFC).

\section{Estudio 1}

\section{Validez de constructo}

Elobjetivode la investigación, era validar el cuestionario ICQ-15 (Bhurmester, Furman, Wittenberg, \& Reis, 1988) con adolescentes. Tras realizar los procesos de traducción, el primer paso, fue estudiar la validez de constructo del cuestionario. 
Tabla 2. Porcentaje de varianza explicado por los cinco factores del ICQ-15

\begin{tabular}{lccc}
\hline Factor & Total & $\begin{array}{c}\text { \% Varianza } \\
\text { explicada }\end{array}$ & $\begin{array}{c}\text { \% Varianza } \\
\text { explicada } \\
\text { acumulada }\end{array}$ \\
\hline Autorrevelación & 2.586 & 17.240 & 17.240 \\
Soporte emocional & 2.538 & 16.923 & 34.162 \\
Aserción negativa & 1.723 & 11.486 & 45.648 \\
Iniciación & 1.619 & 10.792 & 56.440 \\
Gestión de conflictos & 1.267 & 8.449 & 64.888 \\
\hline
\end{tabular}

Tabla 3.Matriz de componentes principales

\begin{tabular}{lccccc}
\hline Ítem & Autorrevelación & $\begin{array}{c}\text { Soporte } \\
\text { emocional }\end{array}$ & $\begin{array}{c}\text { Aserción } \\
\text { negativa }\end{array}$ & Iniciación & $\begin{array}{c}\text { Gestión de } \\
\text { conflictos }\end{array}$ \\
\hline 3 & .694 & & & & \\
5 & .645 & & & & \\
8 & .580 & .814 & & & \\
11 & & .783 & & & \\
14 & & .685 & & & \\
4 & & & .814 & & \\
13 & & & .768 & & \\
12 & & & .673 & .638 & \\
10 & & & & .594 & \\
9 & & & & .549 & \\
7 & & & & & .887 \\
2 & & & & & .749 \\
15 & & & & & .503 \\
1 & & & & \\
6 & & & &
\end{tabular}

Se empleó el método de Componentes Principales con rotación Varimax una vez comprobada la viabilidad del análisis factorial con los siguientes criterios: la matriz de correlaciones presentó un gran número de correlaciones $(87.8 \%)$ con un valor superior a .30 con determinante igual a .002 y el resultado del test de esfericidad de Bartlett mostró que las variables no eran independientes (Prueba Bartlett $=992.725, \quad p$ $<.001)$. El test de Kaiser Meyer Adequacy (KMO) de adecuación de la muestra fue de ,836, indicando que las correlaciones entre parejas de variables pueden ser casi explicadas por las restantes variables. Todos los valores del Measures of Sampling Adequacy (MSA) se encontraban por encima de .81. Estos valores indican que es adecuado realizar un análisis factorial de la matriz de correlaciones. Como se aprecia en la Tabla 2 se obtuvieron cinco factores con eigenvalue mayor que 1 , tomando como criterio asignar un ítem al factor en el que presentara una carga factorial mayor de .40 , que explicaron el $64.88 \%$ de la varianza total.En el factor 1 correspondiente a Autorrevelación se encontró la mayor saturación (17.240\%), seguido del factor 2, Soporte emocional (16.923\%), y los factores Aserción negativa (11.486\%),Iniciación (10.792\%) y Gestión de conflictos $(8.449 \%)$.

La matriz de componentes extraídos mediante el análisis de componentes principales que muestra los factores resultantes y los ítems del cuestionario incluidos en cada uno de ellos, así como sus saturaciones correspondientes. Se refleja la mayor saturación de cada ítem (Tabla 3). Para poder realizar comparaciones, se optó por utilizar el planteamiento que define el ajuste de un modelo a los datos como bueno si la razón entre Chi-cuadrado y los grados de libertad no supera el valor de 3 (Hu \& Bentler, 1999). En la investigación las escalas, mostraron un valor menor de tres, lo que indica su buen ajuste y su validez interna.

\section{Validez convergente}

A continuación, se analizaron los diferentes componentes de los factores, para comprobar la existencia de relaciones significativas entre las dimensiones del cuestionario: Iniciación, Aserción negativa, Soporte emocional, Autorrevelación, Gestión de conflictos. Se calcularon los coeficientes de correlación de Pearson entre los factores del cuestionario ICQ-15 (Tabla 4). 
Tabla 4. Correlaciones de Pearson entre los factores del ICQ-15.

\begin{tabular}{lccccc}
\hline \multicolumn{1}{c}{1} & 2 & 3 & 4 & 5 \\
\hline Iniciación & 1 & & & & \\
Aserción negativa & $.452^{* *}$ & 1 & & & \\
Soporte emocional & $.398^{* *}$ & $.409^{* *}$ & 1 & & \\
Autorrevelación & $.543^{* *}$ & $.388^{* *}$ & $.514^{* *}$ & 1 & \\
Gestión de conflictos & $.385^{* *}$ & $.351^{* *}$ & $.445^{* *}$ & $.336^{* *}$ & 1 \\
\hline$* p<01$ & & & & &
\end{tabular}

Tabla 5. Correlaciones con los factores del BFQ-NA

\begin{tabular}{lccccc}
\hline & Iniciación & Aserción negativa & Autorrevelación & $\begin{array}{c}\text { Soporte } \\
\text { emocional }\end{array}$ & $\begin{array}{c}\text { Gestión } \\
\text { conflictos }\end{array}$ \\
\hline Conciencia & $.27^{* *}$ & $.34 * *$ & $.23^{* *}$ & $.35^{* *}$ & $.38^{* *}$ \\
Apertura & $.28^{* *}$ & $.16^{* *}$ & $.15^{* *}$ & $-.26^{* *}$ & $.11^{* *}$ \\
Extraversión & $.57^{* *}$ & $.31^{* *}$ & $.43^{* *}$ & $.34^{* *}$ & $.24 * *$ \\
Amabilidad & $.14^{* *}$ & -.01 & $.21^{* *}$ & $.23 * *$ & $.36^{* *}$ \\
Inestabilidad & $-.36^{* *}$ & $-.34 * *$ & $-.22 * *$ & $-.27 * *$ & $-.32^{* *}$ \\
emocional & & & & & \\
\hline
\end{tabular}
$* * p<.01$

Tabla 6. Consistencia interna de los factores del ICQ-15

\begin{tabular}{cccccc}
\hline Ítems & Media & DT & $\begin{array}{c}\text { Correlación } \\
\text { ítem-test }\end{array}$ & $\begin{array}{c}\text { Alfa si se } \\
\text { elimina }\end{array}$ & Escala \\
\hline Ítem 1 & 3.583 & 1.109 & $.372^{* *}$ & .844 & \\
2 & 3.665 & 1.120 & $.590 * *$ & .832 & \\
3 & 3.436 & 1.175 & $.436 * *$ & .841 & \\
4 & 4.321 & .904 & $.603 * *$ & .833 & Media = 3.683 \\
5 & 3.807 & 1.187 & $.570^{* *}$ & .833 & Alfa $=.851$ \\
6 & 3.775 & 1.098 & $.431^{* *}$ & .841 & \\
7 & 3.284 & 1.277 & $.458 * *$ & .840 & \\
8 & 3.761 & 1.118 & $.589 * *$ & .832 & \\
9 & 3.468 & 1.195 & $.465 * *$ & .839 & \\
10 & 3.528 & 1.078 & $.333^{* *}$ & .846 & \\
11 & 4.202 & .986 & $.573 * *$ & .834 & \\
12 & 3.417 & 1.150 & $.554 * *$ & .834 & \\
13 & 3.546 & 1.156 & $.479 * *$ & .838 & \\
14 & 4.110 & .944 & $.498 * *$ & .838 & \\
15 & 3.349 & 1.186 & $.276 * *$ & .850 & \\
\hline$* * p<.01$ & & & & &
\end{tabular}

Las dimensiones de ambos instrumentos presentaron correlaciones estadísticamente significativas, las más fuertes $(p \geq .4)$ correspondieron a la extraversión con iniciación $(r=.57)$ y autorrevelación $(r=.43)$. Conciencia y apertura del BFQ-NA presentaron correlaciones con todos los factores del ICQ-15. Por su parte, la amabilidad no correlacionó con la aserción negativa; y la inestabilidad emocional, presentó relación inversa con todos los factores del ICQ-15.

\section{Fiabilidad}

Con el objetivo de comprobar la fiabilidad del instrumento se calculó la consistencia interna de la prueba, mediante el coeficiente de Cronbach, dado que esta escala no está adaptada al español y se quería analizar la adaptación que se realizó para la investigación. Para ello, se obtuvieron los estadísticos (Tabla 6), todos los ítems del cuestionario presentan un alfa de Cronbach superior a .83 y por tanto se puede asumir que los ítems que las forman miden un mismo constructo y que están altamente correlacionados. Este coeficiente, desde el punto de vista de la fiabilidad del cuestionario, se puede calificar de bueno, con valores de .851 en el cuestionario. Los valores por encima de .8 se suelen considerar buenos, lo que indica una gran consistencia interna entre los elementos del cuestionario. 


\section{Estudio 2}

\section{Análisis factorial confirmatorio}

Para el estudio de la estructura interna se utilizó el análisis factorial confirmatorio, que proporciona el marco estadístico adecuado para evaluar la validez y la fiabilidadde cada ítem, en lugar de efectuar sólo valoraciones globales, guiando al investigadorpara que optimice el proceso de construcción o adaptación del cuestionario (Batista \& Coenders, 2000).

En la Figura 1 se muestra el resultado de este análisis factorial confirmatorio (AFC) del modelo generado en el estudio exploratorio, con ecuaciones estructurales mediante el método de extracción de máxima verosimilitud, confirmando la adecuación del modelo ya que se obtuvo un modelo sustentable compuesto por los cinco factores identificados y 15 ítems en total.

Por lo que se refiere al ajuste del modelo, los diversos índices de ajuste resultaron adecuados, por lo que se puede afirmar que el modelo propuesto acerca de la estructura factorial de la escala es sustentable: $\chi^{2}(80)=162.771 ; p<.001 ;$ $\chi^{2} / \mathrm{gl}=2.035 ; \quad$ GFI $=.94 ; \quad$ AGFI $=.96 ; \quad$ CFI $=.936 ;$ $\mathrm{NFI}=.885 ; \mathrm{TLI}=.904 ; \quad \mathrm{RMSEA}=.054$ ， IC $95 \%$ (.042-.065). La estructura factorial se analizó mediante un análisis factorial confirmatorioen el que se planteó un modelo de cinco factores. Esta estructura es a priori, lo quesignifica que los resultados del modelo son plenamente confirmatorios.Los índices mostraron un ajuste del modelo planteado razonablemente adecuado. Así, el índice GFI ofreció un valor de .94 y de 0.96 para el AGFI. El $\chi^{2}$ por su parte ofrecióun valor de 162.771 con 80 grados de libertad. Por último, el RMSEA evaluó el modelo como de óptimo ajuste, siendo su índice de .054 (Lo = $0.042-\mathrm{Hi}=0.065)$. Los resultados obtenidos en estos índices (Figura 1), permiten concluir que el modelo propuesto presenta una aproximación óptima a los datos, y puede contribuir a dar soporte a la hipótesis de la validez del cuestionario.

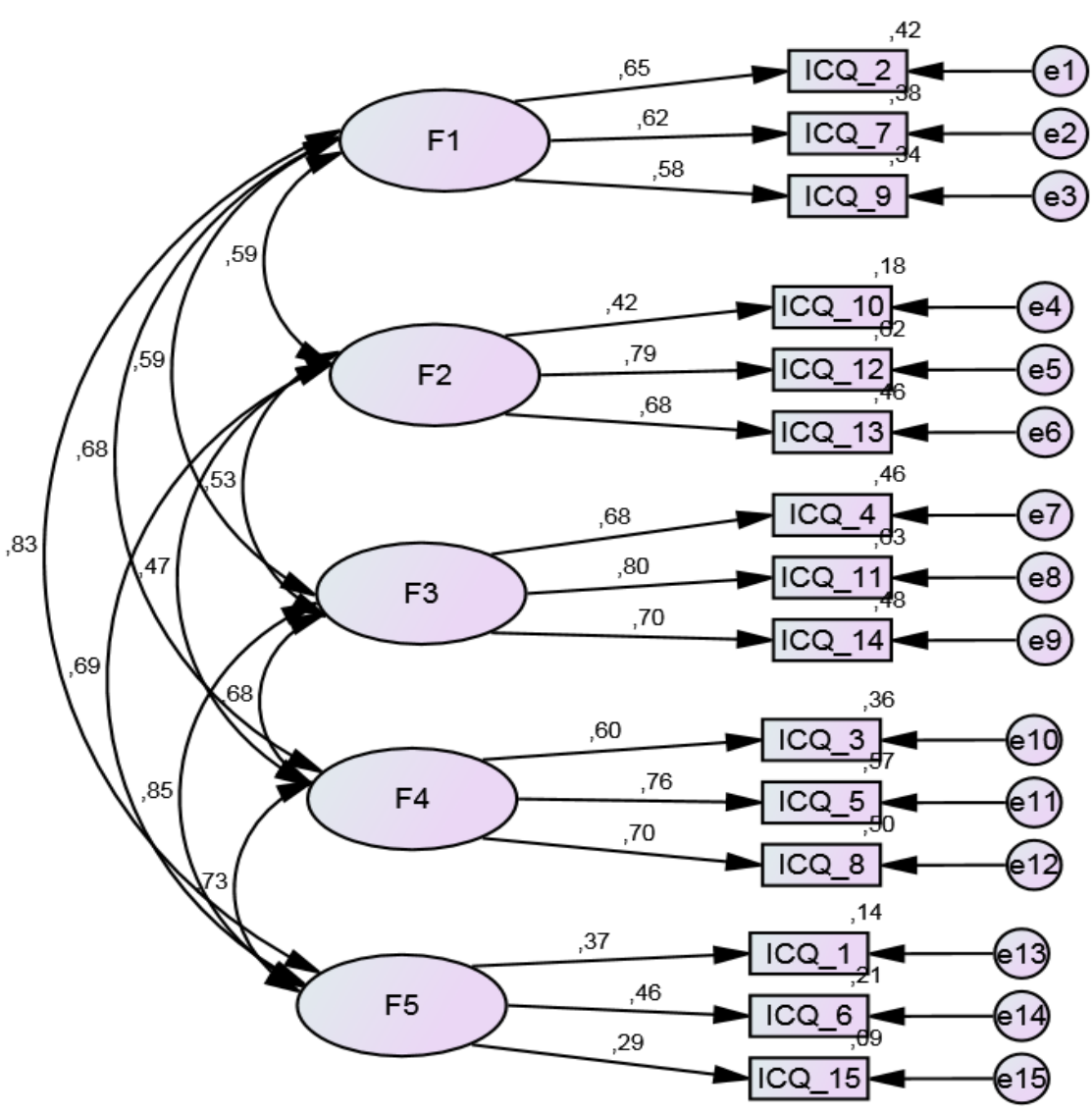

Figura 1. Solución estandarizada del análisis factorial confirmatorio del cuestionario ICQ-15 para la medida de la competencia interpersonal en adolescentes hispanohablantes 


\section{Discusión}

Los objetivos del presente estudio fueron evaluar la posibilidad de utilización del cuestionario de Competencia Interpersonal ICQ15 con adolescentes, como instrumento de evaluación de lacompetencia interpersonal en esas edades, así como su validación para población hispanohablante.

En este estudio se ha validado el cuestionario de competencia interpersonal ICQ-15 con adolescentes, siguiendo un proceso protocolizado de traducción y adaptación y posteriormente analizando sus propiedades psicométricas. Se presentan tanto el procedimiento que se ha realizado para garantizar la equivalencia cultural y lingüística de la escala con su versión original, así como análisis que apoyan la consistencia interna, la validez y la estructura factorial de la misma.

Los principales resultados obtenidos tras realizar el análisis factorial del cuestionario ICQ15 muestran una estructura formada por cinco dimensiones, con una saturación de los ítems elevada, lo que refleja una buena consistencia interna $(, 851)$ del cuestionario, similar a la de trabajos precedentes (Buhrmester et al., 1988; Giromini, de Campora, Brusadelli, D’Onofrio, Zennaro, Zavattini et al., 2015; Górska, 2001; Kanning, 2006).

Los resultados mostraron claramente que los factores del cuestionario ICQ-15 presentan una varianza acumulada del $64.88 \%$. Además se reportan evidencias en favor de la validez en relación con otras variables, ya que por una parte se comprobó que las puntuaciones de los factores del cuestionario ICQ-15 muestran una gran consistencia interna entre los elementos de la escala, lo cual desde el punto de vista de la fiabilidad es excelente.

Además tras explorar la estructura factorial subyacente en el cuestionario decompetencia interpersonal, se ha podido observar que en nuestro estudio se mantienenlos índices de fiabilidad para cada una de las cinco subescalas del cuestionario,ya que los coeficientes de fiabilidad que se obtuvieron en las muestras originalesson similares. Se puede determinar, por consiguiente, que dicha pruebamantiene su consistencia en la valoración de ésta en la adolescencia. Se ha verificadosu estructura de cinco factores, aportándose evidencia empírica de su validez de constructo. Los cinco factores subyacentes se relacionaron significativamente entre sí y las correlaciones más altas se encontraron entre el factor de autorrevelación con los factores de iniciación (,543) y soporte emocional (.514).

Asimismo, al analizar las correlaciones con la prueba de personalidad, se puede concluir que los adolescentes con mayores puntuaciones en extraversión presentan altas puntuaciones eniniciación ( $r=.57)$ y autorrevelación $(r=.43)$. Por su parte, los alumnos que muestran una alta inestabilidad emocional, presentan bajas puntuaciones en competencia interpersonal en todos sus factores, en línea con otros estudios (Nikooyeh, Zarani, \& Fathbadi, 2017). Por tanto, se ha encontrado evidencia preliminar de las relaciones entre el cuestionario ICQ-15y el BFQNA (medida de personalidad) en lo que entendemos como un dato más a favor del cuestionario ICQ-15, que se revela como una herramienta corta, de fácil aplicación, de fácil comprensión y adecuada para medir los constructos que se pretende.

El modelo propuesto para la estructura factorial es sustentable, con cinco factores identificados y quinceítems en total $\left(\chi^{2} / \mathrm{gl}=2.035\right.$; GFI $=.94 ; \quad$ AGFI $=.96 ; \quad$ CFI $=.936 ; \quad$ NFI $=.885 ;$ TLI=.904; RMSEA=.054), por lo que la versión traducida del cuestionario ICQ-15 reveló coeficientes satisfactorios con cada uno de sus cinco factores identificados y con 15 ítems en total en su estructura factorial. Entendemos por lo tanto que estos resultados proporcionan evidencia en favor de la solidez de la estructura de esta medida en adolescentes y revelan que la versión española del cuestionario ICQ-15consigue replicar fielmente la estructura teórica original.

Con los adolescentes, el cuestionario ICQ-15 ha revelado datos satisfactorios ajustándose al modelo teórico subyacente y mostrando alta consistencia interna y validez. Dado que la competencia interpersonal es la capacidad para mantener buenas relaciones con otras personas y esto implica dominar las habilidades sociales básicas, la capacidad para la comunicación efectiva, el respeto, las actitudes prosociales, la asertividad, etc. manteniendo una fuerte relación con las emociones (Bisquerra, 2009; Herzberg \& 
Bräler, 2006; Cook, Gresham, Kern, Barreras, Thornton, \& Crews, 2008), resultará interesante la futura utilización del cuestionario ICQ-15 en relación con estas variables, con estudios más avanzados o incluso para seguir reexaminando sus propiedades.

Estos resultados deben ser interpretados teniendo en cuenta las limitaciones de este estudio, que si bien la muestra es relevante a nivel estadístico, debería ampliarse a otros estratos de la población, dónde podría darse una mayor asociación entre la competencia interpersonal con otros constructos. Serían deseables estudios longitudinales que evalúen la competencia interpersonal y permitan valorar su evolucióna lo largo de un período más largo de tiempo. Además, la competencia interpersonal requiere en muchas ocasiones la posesión de otros tipos de habilidades, por ejemplo, cognitivas, por lo que serían interesantes estudios que evaluasen esta relación. También se podría trabajar este cuestionario con poblaciones de características especiales, como los adolescentes con ansiedad social o autismo, que poseen habilidades sociales más bajas que la población general (Cotugno, 2009; Stravynski, Kyparissis, \& Amado, 2010).

Como principal conclusión, la presente investigación sugiere que la competencia interpersonal en los adolescentes, puede ser valorada por el cuestionario ICQ-15, que se ha mostrado como una medida psicométricamente eficaz, con una buena validez factorial fuerte y una adecuada consistencia interna; además de ser una herramienta breve y fácil de administrar. Aunque es necesario un mayor número de estudios e investigaciones en este sentido, los hallazgos señalan el ICQ-15 como una herramienta útil para la medida de la competencia interpersonal en los adolescentes.

\section{Referencias}

Barbaranelli, C., Carpara, G., Rabasca, A., \& Pastorelli, C. (2003). A questionnaire for measuring the Big Five in late Childhood. Personality and Individual Differences, 34, 645-664.

Batista, J. M., \& Coenders, G. (2000). Modelos de ecuaciones estructurales, Madrid: La Muralla.

Bisquerra, R. (2009). Psicopedagogía de las emociones. Madrid: Síntesis.

Buhrmester, D., Furman, W., Wittenberg, M. T., \& Reis, H. T. (1988). Fivedomains of interpersonal competence in peer relationships. Journal of Personality and Social Psychology, 55, 991-1008. doi: 10.1037/0022-3514.55.6.991

Caballo, V. (2002). Manual de evaluación y entrenamiento de las habilidades sociales. Madrid: Siglo XXI.

Caballo, V., Salazar, I., \& Equipo CISO-AEspaña (2017). Desarrollo y validación de un nuevo instrumento para la evaluación de las habilidades sociales: El "Cuestionario de habilidades sociales" (CHASO). Behavioral Psychology / Psicología Conductual, 25(1), 524.

Cook, C. R., Gresham, F. M., Kern, L., Barreras, R. B., Thornton, S., \& Crews, S. D. (2008). Social skills training forsecondary students with emotional and/or behavioral disorders: A review and analysis of the meta-analytic literature. Journal of Emotional and Behavioral Disorders, 16, 131-144. doi: 10.1177/1063426608314541

Coroiu, A., Meyer, A., Gomez-Garibello, C.A., Brähler, E., Hessel, A., \& Körner, A. (2015). Briefform of the Interpersonal Competence Questionnaire (ICQ-15). Development and preliminary validation with a German populationsample. European Journal of Psychological Assessment, 31(4), 272-279. doi: 10.1027/1015-5759/a000234

Cotugno, A. J. (2009). Social competence and social skills training and intervention for children with autism spectrum disorders. Journal of Autism and Developmental Disorders, 39, 1268-1277. doi: 10.1007/s10803-009-0741-4

Del Barrio, M. V., Carrasco, M. A., \& Holgado, F.P. (2006). BFQ-NA cuestionario de los Cinco Grandes para niños y adolescentes (adaptación a la población española). Madrid: TEA Ediciones. (2006).

Ferriter, C., Eberhart, N. K., \& Hammen, C. (2010). Depressive symptoms and social functioning in peer relationships as predictors of eating pathology in the transition to adulthood. Journal of Social and Clinical Psychology, 29, 202-227. 
doi: 10.1521/jscp.2010.29.2.202

Gálvez-Nieto, J. L., Polanco, K., \& Salvo, S. (2017). Propiedades psicométricas de la Escala de Autoconcepto Académico (EAA) en estudiantes chilenos. Revista Iberoamericana de Diagnóstico y Evaluación - e Avaliação Psicológica, 43(1), 5-16. doi: 10.21865/RIDEP43_5

Giromini, L., de Campora, G., Brusadelli, E., D’Onofrio, E., Zennaro, A., Zavattini, G. C., \& Lang, M. (2015). Validity and reliability of the Interpersonal Competence Questionnaire: Empirical evidence fromanItalian study. Journal of Psychopathology and Behavioral Assessment, 38(1), 113-123. doi: 10.10007/s10862-015-9499-5

Górska, M. (2011). Psychometric properties of the polish version of the Interpersonal Competence Questionnaire (ICQ-R). European Journal of Psychological Assessment, 27(3), 186-192. doi: 10.1027/1015-5759/a000066

Herzberg, D. S., Hammen, C., Burge, D., Daley, S.E., Davila, J., \& Lindberg, N. (1998). Social competence as a predictor of chronic interpersonal stress. Personal Relationships, 5, 207-218. doi: 10.1111/j.1475-6811.1998.tb00168.x

Herzberg, P.Y., \& Brähler, E. (2006). Assessing the Big-Five personality domainsvia short forms: A cautionary note and a proposal. European Journal of Psychological Assessment, 22, 139-148. doi: 10.1027/10155759.22.3.139

Holopainen, L., Lappalainen, K., Junttila, N., \& Savolainen, H. (2011). The role of social competence in the Psychological well-being of adolescents in secondary education. Scandinavian Journal of Educational Research, 56, 199-212. doi: 10.1080/00313831.2011.581683

Hu, L., \& Bentler, P. M. (1999). Cut off criteria for fit indexes in covariance structure analysis: Conventional criteria versus new alternatives. Structural Equation Modeling: A Multidisciplinary Journal, 6, 1-55. doi: 10.1080/10705519909540118

Kanning, U. P. (2006). Development and validation of a German-languageversion of the Interpersonal Competence Questionnaire
(ICQ). European Journal of Psychological Assessment, 22, 43-51. doi: 10.1027/10155759.22.1.43

Kinnaman, J. E. S. \& Bellack, A. S. (2012). Social Skills, in O'Donohue, W., \& Fisher, J. E. (eds.) Cognitive Behavior Therapy: Core principles for practice. Nevada: John Wiley and Sons.

Körner, A., Drapeau, M., \& Geyer, M. (2006). The association between interpersonal relationships and health in a representativepopulationsample. Archives of Psychiatry and Psychotherapy, 8, 5-12.

Montero, I. \& León, O. (2007). A guide for naming research studies in Psychology. International Journal of Clinical and Health Psychology, 7(3), 847-862.

Muñiz, J., Elosua, P., \&Hambleton, R.K. (2013). International Test Commission. Guidelines for test translation and adaptation. 2nd Ed. doi: 10.7334/psicothema/2013.24

Muralidharan, A., Sheets, E. S., Madsen, J., Craighead, L. W., \& Craighead, W. E. (2011). Interpersonal competenceacrossdomains: Relevanceto personality pathology. Journal of Personality Disorders, 25, 16-27. doi: 10.1521/pedi.2011.25.1.16

Navarro, G., Varas, M., \& Maluenda, J. (2017). Propiedades psicométricas del Inventario de Asertividad de Gambrill y Richey en estudiantes universitarios chilenos. Revista Iberoamericana de Diagnóstico y Evaluación - e Psicológica, 43(1), 33-43. doi: 10.21865/RIDEP43_33

Nikooyeh, E., Zarani, F., \& Fathabadi, J. (2017). The mediating role of social skills and sensationseeking in the relationship between trait emotional intelligence and schooladjustment in adolescents. Journal of Adolescence, 59, 45-50. doi: 10.1016/j.adolescence.2017.05.012

Parade, S. H., Leerkes, E.M., \& Blankson, A. N. (2010). Attachment to parents, social anxiety, and close relationships of female students over the transition to college. Journal of Youth and Adolescence, 39, 82-92.

doi: 10.1007/s10964-009-9396-x

Segrin, C. (2001). Interpersonal processes in psychological problems. New York, NY: The GuilfordPress. 
Stravynski, A., Kyparissis, A., \& Amado, D. (2010). Social phobia as a deficit in social skills. In G. Hoffmann \& P. M. DiBartolo (Eds.), Social anxiety: Clinical, developmental, and social perspectives(2nd. ed.). London, UK: Elsevier. 